\title{
PRIMARY PULMONARY TUBERCULOSIS IN CHILDREN 1
}

\author{
By R. G. CANTI
}

(From the Pathological Laboratory, St. Bartholomew's Hospital)

IN 1916 Barty King (1) published a translation of A. Ghon's Die primare Lungenherd bei der Tuberlulose der Kinder.

Kuss, E. Albrecht, H. Albrecht, Ghon, and others belong to a school which holds that there is a special form of tuberculosis in children, consisting of a primary lung focus, caused by inhalation of tubercle bacilli. This foous is responsible for the greater number of deaths from tuberculosis in children, and, as a rule, escapes clinical diagnosis.

Ghon performed autopsies on 644 cases, of which 184 (28.57 per cent.) were tuberculous, and of these 170 (92.40 per cent.) showed what he believes to be, a primary focus of infection in the lung.

The present work was undertaken with a view to repeating these observations. The cases dealt with form a continuous series of eighty-four autopsies in children up to 10 years of age, carried out by the writer at St. Bartholomew's Hospital. The series is not entirely free from involuntary selection, owing to the presence of an abmormally large proportion of children under one year of age (thirty-three cases or 39.30 per cent.), and the absence of cases of bone and joint disease, most of which find their way to special hospitals.

According to Ghon the lung focus is usually small, and varies from the size of a pin's head to that of a cherry ; most often it is about the size of a pea. It is usually single (72.35 per cent.), and only on rare occasions are more than two or three foci found. The focus is presumed to have origin in the aggregations of lymphoid tissue found in the neighbourhood of the small bronchi, the evidence brought forward in support of this view being that a fissure, which is thought to be the remains of a small bronchus, is frequently found in the middle of a focus, and further, that on tracing distally along a bronchus a dead end is frequently reached which is found to be within a focus, and that the tissue beyond such a focus is oftentimes atelectatic.

The focus, commencing as a small tubercle, soon increases in size and involves

1 This work was carried out under the auspices of the Medical Research Committee, from whom a grant was received.

[Q.J.M. Oct., 1919.] 
the neighbouring structures. Around it small anatomical tubercles may frequently be seen, and fibrous tissue may be formed in an attempt at encapsulation. In the larger foci caseation usually takes place, which is hard and dry rather than soft or liquid, but occasionally a cavity is formed. The process may be arrested during any of these stages, as evidenced by fibrosis or calcification.

The situation of the focus is usually immediately beneath the pleura, which is frequently involved. The right lung is more often affected than the left (3:2), and the order of frequency with which the various lobes are involved, in cases where only one lung focus is found, is as follows :

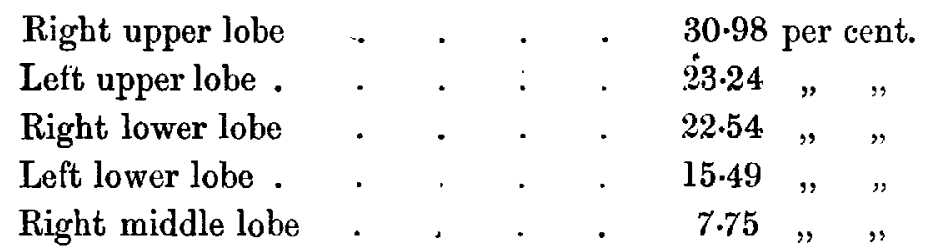

Kuss, however, states that the lower lobes are more frequently affected than the upper.

Lesions are scarcely ever found at the apices, the commonest site in the upper lobes being the middle of the anterior surface. In the lower lobes the posterior surface near the lower edge is most frequently affected.

The mediastinal glands are classified by Ghon in four groups :

\begin{tabular}{|c|c|c|c|}
\hline Superior tracheo-bronchia & . & & $\begin{array}{l}\text { Those in the angle between the } \\
\text { trachea and bronchus on either } \\
\text { side. }\end{array}$ \\
\hline Inferior tracheo-bronchial & . & & $\begin{array}{l}\text { Those in the bifurcation of the } \\
\text { trachea. }\end{array}$ \\
\hline Paratracheal & . & . & $\begin{array}{l}\text { Those forming a chain on either } \\
\text { side of the trachea. }\end{array}$ \\
\hline Broncho-pulmonary . & . & . & $\begin{array}{l}\text { Those at the hilus of the lung } \\
\text { and between the branches of } \\
\text { the main bronchi. }\end{array}$ \\
\hline
\end{tabular}

From anatomical observations it has been found that the superficial and deep lymphatic vessels dischargo their contents, first into the broncho-pulmonary glands, and then into one of the remaining groups, which are closely connected together by anastomosing lymphatic vessels. For the most part the lymphatic vessels from the middle and lower portions of the lung discharge their contents into the inferior tracheo-bronchial glands, and those from the upper lobes into the superior tracheo-bronchial glands, whence crossing over the middle line frequently takes place.

Two facts of importance are found with regard to the relation of the lung focus to the mediastinal glands; the first is that if there is a lung focus there are also tuberculous glands in the mediastinum (Ghon has reported one case in 170 in which this was not so); the second is that if the glands are affected a tuberculous focus in the lung is present (Ghon has reported seven cases showing 
tuberculosis of the glands of the mediastinum with no evidence of a source of infection outside the lung, in which he was unable to find a lung focus).

The glands exhibit the familiar lesions of tuberculous lymphatic glands. If their apparent age be compared with that of the lung focus it is found that either the lung focus is older or of the same age. It never appears more recent.

With regard to the relation between the glands and the lung focus it is found that the infection of the glands follows the course which might be expected from a knowledge of the anatomical arrangement of the mediastinal lymphatic system. A focus, for example, in the right upper lobe will be found to be accompanied by lesions in the broncho-pulmonary glands, especially those in relation to the eparterial bronchus. These lesions will not be extensive, as the initial size of the glands is not great. The infection, having passed this barrier, will reach the groups of glands in relation to the main bronchus and the trachea. Of these groups the right superior tracbeo-bronchial will be the first and the most severely affected. Owing to intimate anastomosis the infection may spread to neighbouring groups, first to the right paratracheal and the inferior tracheo-bronchial, and later to the left superior tracheo-bronchial group. In like manner a lesion in any other situation in the lung will be found to be accompanied by a similar spreading out of the glands in the form of a fan or segment of a circle, the focus being found at the handle of the fan, or centre of the circle.

Thus, if the distribution of affected lymphatic glands in the mediastinum be examined, it is usually possible to predict with certainty the situation of the lung focus, and, as a rule, no difficulty is experienced in detecting the focus within a few seconds by palpating and sectioning the suspected lobe.

As the disease progresses fresh lesions occur, which must be carefully distinguished from the original focus. Important amongst these are tuberculous broncho-pneumonia, due to discharge of material from the focus or perforation of a bronchus by an adherent gland; the adhesion of a gland to the lung tissue and the resulting spread of the lesion by direct extension; and a haematogenous infection resulting in miliary tuberculosis with or without broncho-pneumonia.

In order to establish the priority of the lung focus it is necessary to show, first, that it is older than the lesions in the mediastinal glands, and, secondly, that it is older than any other focus which may be present in the body.

It is held by many that the disease is primary in the mediastinal glands, the reason put forward being either that the glandular tuberculosis is the only disease seen at autopsy, or that, if a focus is present in the lung, it is much smaller than the affected glands, and therefore secondary to them.

Against these arguments Ghon puts forward the following :

(1) On studying the distribution of affected glands it is found, from a knowledge of the anatomy of the lymphatic system of the lungs and mediastinum, that those glands are tuberculous which one would expect to find tuberculous if the lung focus were primary.

(2) The changes in the glands always appear of more recent date than, or at least only of the same age as, the lung focus. They never appear older. 
(3) Tuberculous glands in the mediastinum are hardly ever found without a corresponding lesion in the lung.

The hypothesis of a primary lung focus becomes even more tenable if the propagation of the disease in the reverse direction be considered. If this unlikely theory be correct, the disease, having commenced and spread in the mediastinal glands, must arbitrarily have selected a point in the lung more or less remote from the infected glands for the deposition of a single secondary focus, leaving the intervening lung tissue free from tuberculosis.

Again, the argument that the lung focus is not primary because it is smaller than the gland lesion does not hold good, for it must be remembered that glands are organs designed to hypertrophy to many times their normal size in order to cope with infection, whereas no such mechanism is present in other tissues, where the reaction is local, and is set up for the purpose of limiting or reducing the size of the lesion.

As a preliminary step in proving that the disease may reach the lung by metastasis from some other focus in the body, it would be necessary first to establish the presence of such a focus, and, secondly, to demonstrate that it is older than the focus in the lung. But Ghon has shown that only in 8.87 per cent. of cases could a focus elsewhere be shown to be of the same age as the lung focus, and in no case was there evidence that it was of greater age. Further, in a disease of the nature of tuberculosis, it would be expected that metastases would be multiple in a large proportion of cases, whereas in his series 72.35 per cent. of the cases showed only one lung focus.

The question as to the channel of infection of thoracic tuberculosis in children has been investigated from quite a different point of view by Eastwood, F. Griffith, and A. S. Griffith, who studied the types of tubercle bacilli found in various lesions. Bearing in mind that an air-borne infection is derived almost entirely from the sputum of phthisical patients, and that these are infected for the most part with the human type of tubercle bacillus, and that ingestion tuberculosis is derived almost entirely from milk infected with the bovine typo of bacillus, it is reasonable to suppose that if the organism in thoracic tuberculosis in children were due to inhalation the human type of bacillus would be present, and that if the thoracic lesion were metastatic the organism isolated would be of the type most commonly found in connexion with intestinal lesions.

After selecting cases where there was strong anatomical evidence in favour of the lesion having arisen in the respiratory tract, Eastwood and F. Griffith (2) found forty-three cases yield the human type of tubercle bacillus out of forty-four cases from which tubercle bacilli were cultivated, and A. S. Griffith (2) found all of twenty-two cases yield the human type of tubercle bacillus. When, however, cases were selected where there was strong anatomical evidence that the infection was alimentary in origin, Eastwood and F. Griffith found that twelve out of fifteen cases, and A. S. Griffith six out of eight cases, yielded the bovine type of tubercle bacillus.

In the present series of eighty-four cases, sixteen (19.05 per cent.) showed 
tuberculous lesions. The following are short notes of these cases, together with diagrams, compiled from dissection of the thoracic contents :

I. Cases showing Foci in the Lungs.

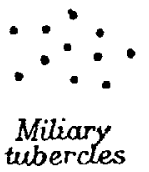

Miliary
tubercles

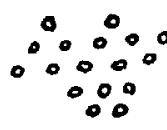

Caseating

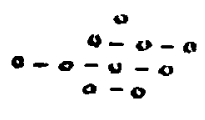

Tuberculous broncho-pneumonia.

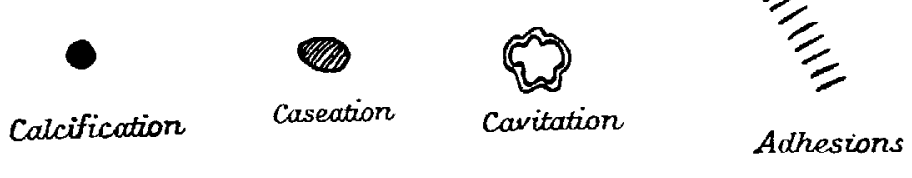

Key to Diagrams.

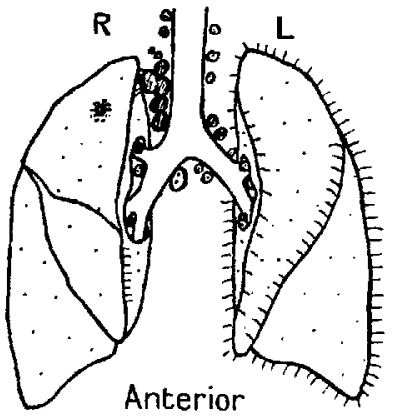

FIG. 1.

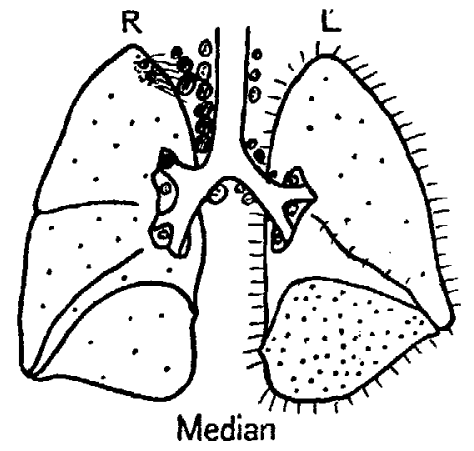

Fig. 2 .

Case I. Figs. 1 and 2. Male, aged 6 years. Cause of death : tuberculous meningitis.

A liquefying caseous focus the size of a large pea, surrounded by a zone of large tubercles and situated immediately beneath the middle of the anterior surface of the right upper lobe. Two or three small fibro-caseous foci surrounded by confluent tubercles situated at the right apex. Large miliary tubercles in moderate numbers throughout the lung, especially on the diaphragmatic surface of the left side. Adhesions all over the left pleura and mediastinal surface of the right middle lobe. Adhesions between right apex and neighbouring glands. A few small right paratracheal glands, the lower ones caseous and the upper showing anatomical tubercles. A chain of small left paratracheal glands showing small tubercles. Mass of about four caseous right superior tracheo-bronchial glands, the largest the size of a cob-nut. A few small caseous left superior tracheo-bronchial glands with anatomical tubercles, the largest on the right side. One small caseous gland in relation to the eparterial bronchus, and several other small broncho-pulmonary glands on either side, showing miliary tubercles. Generalized tuberculosis, tuberculous meningitis.

Case II. Fig. 3. Female, aged 7 years. Cause of death: tuberculous meningitis:

Calcareous focus, the size of a hemp-seed, situated in the middle of lower anterior edge of the right upper lobe. Adhesions all over the right side. One 
caseous right paratracheal gland the size of a cob-nut, one small caseous right superior tracheo-bronchial gland the size of a small pea, one small caseous broncho-pulmonary gland below the eparterial bronchus. Caseous tuberculoma of the cerebellum, tuberculous meningitis. One small calcareous tubercle of spleen.

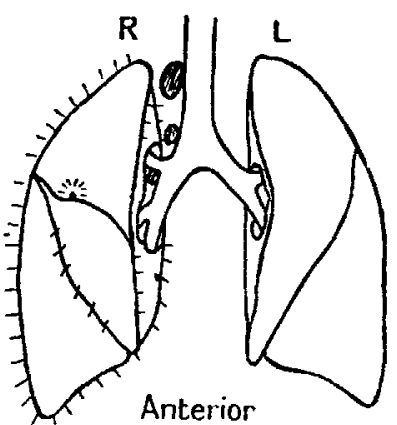

Fict. 3.

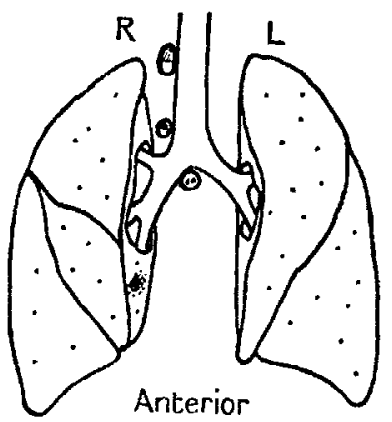

FIG. 4.

Case III. Fig. 4. Female, aged 6 years. Cause of death: tuberculous meningitis.

Focus the size and shape of half a split pea, consisting of a mass of confluent caseous tubercles situated on the mediastinal surface of the right middle lobe and involving the pleura. Moderate sprinkling of grey miliary tubercles throughout both lungs. One semi-caseous right paratracheal gland the size of a large cob-nut. One small partly caseous right superior tracheo-bronchial gland. One small inferior tracheo-bronchial gland, with miliary tubercles, situated in the middle line. A few fair-sized caseous tubercles of kidney. A few miliary tubercles in liver.

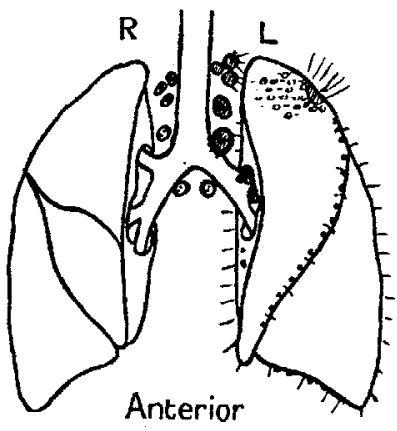

FIG. 5.

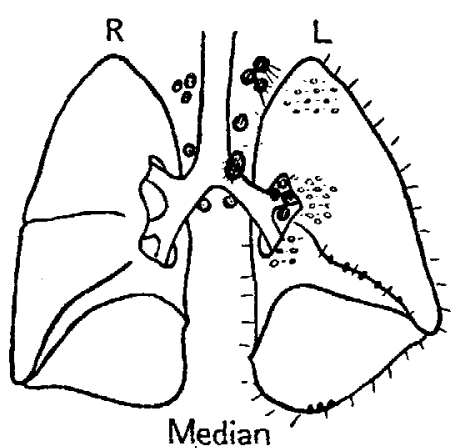

Fra. 6.

Case IV. Figs, 5 and 6. Female, aged $4 \frac{1}{2}$ years. Cause of death : tuberculous meningitis.

Caseating focus, the size of a large cob-nut, situated in the left upper lobe somewhat anterior to the mid-axillary line, one inch below the apex, and involving the pleura between the lobes as well as on the surface of the lung. Large miliary tubercles and tuberculous broncho-pneumonia involving the upper portion of the left upper lobe, except at the extreme apex. Large miliaxy tubercles and tuberculous broncho-pnemmonia at the root of the left lung. Adhesions all over left pleura. Large miliary tubercles in pleura, especially between the lobes, at the anterior edge of the lung, and on surface opposed to pericardium. Many caseous left paratracheal glands, the largest the size of an almond, some adherent to the apex. Many medium-sized semi-caseous right paratracheal glands. Large caseous right superior tracheo-bronchial gland firmly adherent to bronchus. Medium-sized left superior tracheo-bronchial gland with 
anatomical tubercles. Two small inferior tracheo-bronchial glands with anatomical tubercles. Several small caseous left broncho-pulmonary glands situated about the superior bronchus. Tuberculous meningitis. Large tubercles of spleen, diaphragm, and pericardium.

Case V. Fig. 7. Female, aged $1 \frac{1}{2}$ years. Cause of death: tuberculous meningitis.

A caseous focus, the size of a large pea, situated on the inner surface of the left upper lobe a short distance below the apex, with a few large miliary tubercles in the neighbourhood. Tuberculous broncho-pneumonia of the upper portion of left upper lobe. Scanty miliary tubercles throughout both lungs. Adhesions between upper and lower lobes on the left side and upper and middle lobes of right side. Many moderate-sized caseous left paratracheal glands. A few small caseous right paratracheal glands. A large group of moderate-sized caseous left superior tracheo-bronchial glands surrounding the aorta and adherent to the lung. One small caseous inferior tracheo-bronchial gland on either side. Several mediumsized caseous left broncho-pulmonary glands. Generalized tuberculosis, tuberculous meningitis.

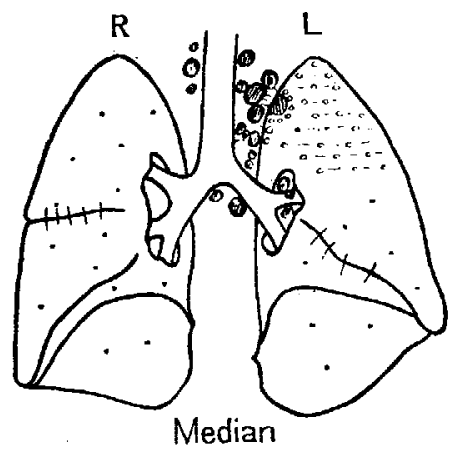

Fig. 7 .

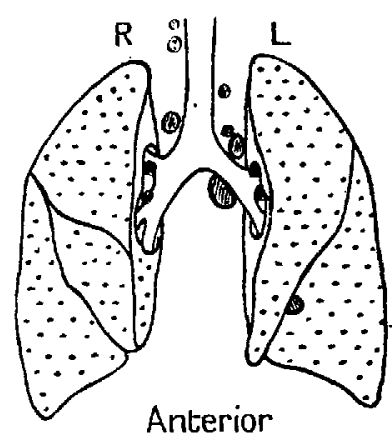

FIG. 8 .

Case VI. Fig. 8. Female, aged 6 months. Cause of death: tuberculous meningitis.

A caseous focus, the size of a large pea, situated on the interlobar edge of the left lower lobe in front. Large miliary tubercles, in many places confluent, throughout both lungs. Two right paratracheal glands the size of large peas, one caseous, the other showing anatomical tubercles. One caseous left paratracheal gland. One caseous right superior tracheo-bronchial gland, the size of a cob-nut. Two smaller left superior tracheo-bronchial glands. One liquefying inferior tracheo-bronchial gland, the size of a large cob-nut, more inclined to the left side than the right. A few small tuberculous uleers, involving, but not exceeding, Peyer's patches, situated about the middle of the small intestine. Caseous mesenteric glands of the average size of a large pea. Generalized tuberculosis, tuberculous meningitis.

Case VII. Fig. 9. Female, aged $7 \frac{1}{2}$ years. Cause of death: tuberculous meningitis.

A caseous focus, the size of a large pea, surrounded by small tubercles, about $1 \frac{1}{2}$ inches above the lower posterior border of the left lower lobe and adherent to the pleura. A few scattered miliary tubercles throughout. A few small left paratracheal glands showing tubercles, of which some are confluent and caseous. Caseous left superior tracheo-bronchial gland, the size of a raisin. Many caseous inferior tracheo-bronchial glands, the largest the size of a raisin, and situated on the left side. Several left broncho-pulmonary glands with confluent caseous tubercles. Tuberculous meningitis. 
Case VIII. Fig. 10. Male, aged $1 \frac{1}{2}$ years. Cause of death : meningococcal meningitis.

A fibro-caseating focus, the size of a cherry, situated about the middle of the diaphragmatic surface of the left lower lobe. Adhesion to the diaphragm over the region of the lung focus. A few caseating left superior tracheo-bronchial glands, the largest as big as an orange pip. Two caseous inferior tracheobronchial glands situated on the left side, the largest the size of a cob-nut. A large crop of discrete left broncho-pulmonary glands occupying a space the size of a walnut. None of these glands was larger than a large pea. Their cut surface showed confluent tubercles. A few small miliary tubercles of the liver and spleen.

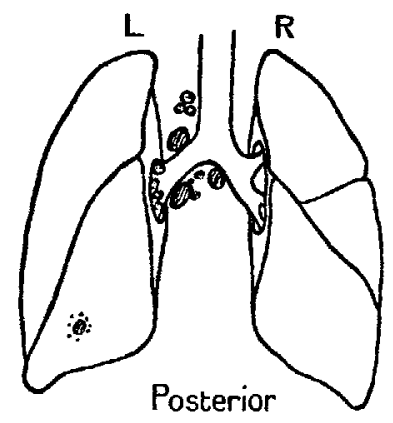

FIG. 9.

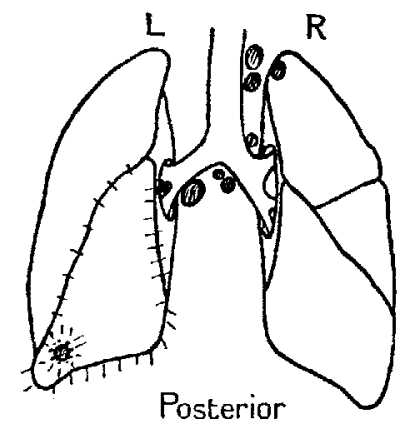

FIG. 11.

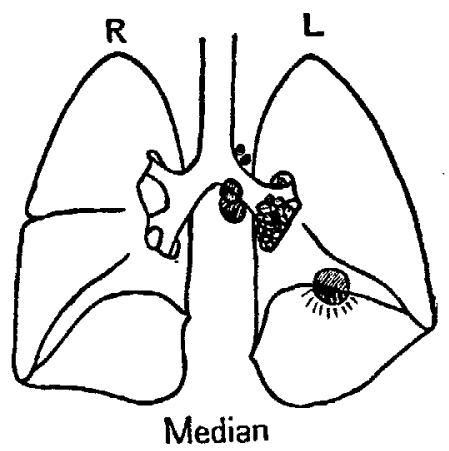

FIG. 10.

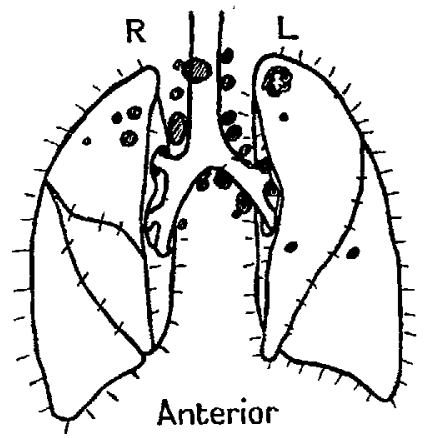

FIG. 12.

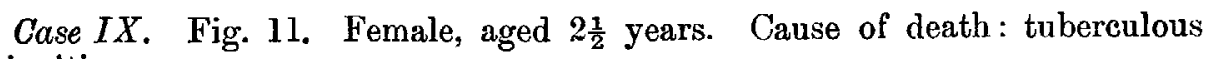
meningitis.

A caseous focus, the size of a pea, with tubercles surrounding, situated near the inferior edge of the left lower lobe behind and involving the pleura. Adbesions all over the external and interlobar surfaces of the left lower lobe. A caseous focus on the inner side of the right apex. Two large caseous right paratracheal glands. One small caseous right superior tracheo-bronchial gland. A few small caseous inferior tracheo-bronchial glands situated near the middle line, and one large gland situated on the left side, adherent to the left bronchus. One moderate-sized caseous left broncho-pulmonary gland between the main branches of the bronchus. Enlarged glands in the right side of the neck. Generalized tuberculosis, tuberculous meningitis.

Case X. Fig. 12. Male, aged $4 \frac{1}{2}$ years. Cause of death: tuberculous cachexia.

Cavity the size of a small cob-nut, irregular in shape, lining shaggy, surrounded with fibrous tissue, situated about the middle of the left apex. Two other foci, small and caseous, present in upper left lobe. One small caseous focus in lower left lobe in front near superior edge. Four foci, varying from the size of a hemp- 
seed to that of a pea, the three largest caseous, present in the upper portion of the right upper lobe, three in front and three behind. All foci near the surface of the lung. Firm adhesions all over both lungs and between their lobes. Several caseous right paratracheal glands, the largest the size of a small cob-nut, lying somewhat in front of the trachea. Chain of small caseous left paratracheal glands. One caseous right superior tracheo-bronchial gland the size of a large cob-nut. Several small caseous left superior tracheo-bronchial glands. Several caseous inferior tracheo-bronchial glands, the largest, the size of a cherry, being on the left side. A few small caseous broncho-pulmonary glands on either side. Enlarged glands in neck, axillac, and groins. Tuberculous ulceration of colon. Numerous caseating mesenteric glands. Tuberculous focus in the left kidney the size of a hemp-seed. A few small anatomical tubercles in liver.

\section{Cases showing no Lung Foci (exclusive of Miliary Tuberculosis).} jaundice.

Case X1. Fig. 13. Female, aged 7 years. Cause of death: purpura with

Pleural adhesions at left apex, and at right base in the mid-axillary line. One left paratracheal and one left superior tracheo-bronchial gland, both fibrocaseous. Several caseous mesenteric glands.

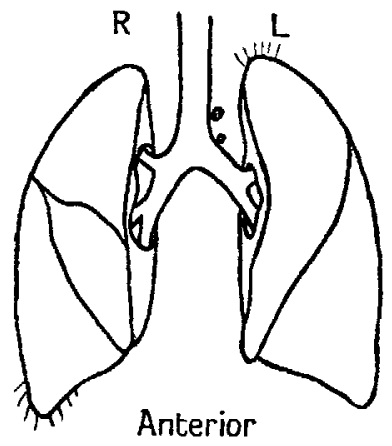

Fig. 13.

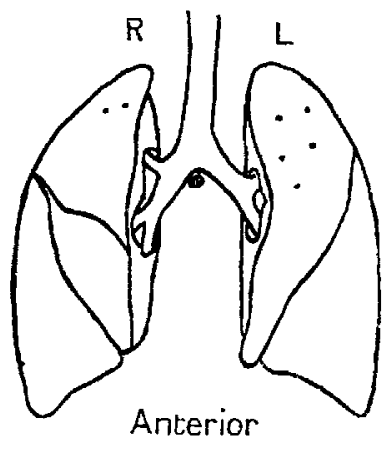

FIG. 14.

Case XII. Fig. 14. Female, aged 3 months. Cause of death: tuberculous meningitis.

Large, but scanty, miliary tubereles on surface of, and throughout, both upper lobes, one or two in left lower lobe. Small caseous inferior tracheo-bronchial gland in the middle line. Tuberculous peritonitis with considerable matting of intestines. Caseous mesenteric glands.

Case XIII. Male, aged 4 years. Cause of death: generalized tuberculosis.

Large and closely-set miliary tubercles throughout both lungs. Bronchopulmonary and mediastinal glands enlarged and containing miliary tubercles. Two tuberculous ulcers in ileum. Tuberculous peritonitis. Matted mass of fibro-caseous mesenteric glands as big as fist. Generalized tuberculosis.

Case XIV. Male, aged 9 years. Cause of death: tubereulous peritonitis. Fair number of tubercles on visceral pleurae. 'Tuberculous peritonitis, with large numbers of tubercles over the peritoneum, and much adhesion. Moderate numbers of caseous mesenteric glands.

Case $X V$. Male, aged $6 \frac{2}{2}$ years. Cause of death : lymphatic leukaemia.

A few caseous mesenteric glands.

Case XVI. Female, aged 10 weeks. Cause of death : 'marasmus.'

Small anatomical tubercles, in large numbers, in mucous membrane of colon. (These were shown to contain giant cell systems and tubercle bacilli.) 
The lung focus in this series was found to be most often the size of a pea. In one (Case II) it was small and difficult to find. The largest focus found was the size of a cherry, and occurred in a case (Case VIII) which died from meningococcal meningitis.

Single lung foci were found on eight occasions (Cases I-VIII). Two foci, apparently about the same age, were found in one (Case IX), and one (Case X) showed more than two foci, of which one, being cavernous, appeared decidedly older than the remainder.

In the majority of cases the focus was caseous or fibro-caseous. In one (Case II) it was calcareous, in one (Case I) liquefying, and in one (Case X) there was cavitation.

In all cases the lung focus was situated just beneath or involving the pleura. In the cases where only one lung focus was present it was situated in the left lower lobe in three cases, in the right upper lobe in two, in the left upper lobe in two, and in the right middle lobe in one.

Tuberculosis of the mediastinal glands, other than acute miliary tuberculosis, was found in twelve cases. In ten cases these glands were in anatomical relation to a lung focus in the manner already described, and in two (Cases XI and XII) no lung focus could be found. 'In each of these two cases the lesions in the glands were of small extent, and extensive abdominal tubereulosis was present. In all cases the most severely affected glands were caseous or fibro-caseous.

Tuberculous lesions of the intestines were found in four cases. In two (Cases VI and XIII) there was ulceration of Peyer's patches; in one (Case X) there was extensive ulceration of the colon; and in one (Case XVI) there were numerous small anatomical tubercles situated in the mucous membrane of the colon.

Tuberculous mesenteric glands were present in seven cases. They were present in three of the cases where tuberculosis of the intestines was found (Cases VI, X, and XIII), and in four cases in which neither tuberculosis of the intestine nor of the lung could be discovered.

In reviewing these cases as a whole it is found that ten (62.50 per cent. of all cases of tuberculosis) showed foci in the lung, and of these eight (80 per cent.) showed only one focus. Seven of these ten cases with lung foci showed no tuberculous lesions outside the thorax; threo (Cases VI, IX, and X) showed lesions in or connexion with the alimentary tract. Of the remaining six cases in which no lung focus was found all showed abdominal tuberculosis. 1

Broncho-pneumonic changes in the lung tissue, due apparently to the direct spread from a tuberculous lesion, were found in three cases. One (Case V) showed the change in the immediate neighbourhood of the lung focus; one (Case I) showed broncho-pneumonia with small foci of caseation, at the apex and in direct relation to an adherent tuberculous paratracheal gland; and one (Case IV, Fig. 6) showed tuberculous broncho-pneumonia both at the hilus,

1 It is noteworthy that this series contains a larger proportion of cases of abdominal tuberculosis than reported by Ghon. 
spreading apparently from the broncho-pulmonary glands, and at the apex, in the neighbourhood of the lung focus.

Miliary tuberculosis was found in five cases where a lung focus was present, and in two cases where no lung focus could be found.

From the foregoing it would therefore appear highly probable that tuberculosis of the mediastinal glands is secondary to a focus in the iung, and, further; that the focus in the lung is primary and not secondary to a focus elsewhere. For if the incidence of the primary lung focus be not accepted we are faced with the following anomaly, namely, that if the lung focus is metastatic, then it is at least curious that cases with a lung focus usually show no other evidence of a possible portal of entry, and, conversely, that where there is evidence that tubercle bacilli may have entered through some other organ, then it is uncommon to find a lung focus. If this anomaly be admitted there remains the argument that a lung focus is a metastasis from a focus elsewhere, and that only when this focus is undiscoverable does a metastasis in the lung occur. And this is manifestly absurd.

\section{Conclusion.}

The findings in this series agree substantially with those of Ghon. Any differences which may exist are numerical rather than qualitative, and are no greater than might reasonably be expected considering the difference of the conditions under which the observations in the two series were made.

The outstanding features are as follows:

The almost constant finding of a lung focus when tuberculous mediastinal glands are present, and the close anatomical relation of those glands to the lung focus.

The frequent singleness of the lung focus.

The constant finding of tuberculous mediastinal glands when a lung focus is present.

The almost constant absence of a lung focus when the portal of entry appears to be elsewhere.

The almost constant absence of evidence that the portal of entry might be elsewhere when a lung focus is present.

These observations, especially when taken in conjunction with the bacteriological findings of Eastwood, F. Griffich, and A. S. Griffith as to the distribution of human and bovine tubercle bacilli, constitute strong evidence in favour of the common existence of primary pulmonary tuberculosis in children.

\section{REFERENCES,}

1. Ghon, A., The Primary Lung Focus of Tuberculosis in Children, transl. by D. Barty King, Lond., 1916, 8vo.

2. Eastwood, A., Griffith, F., and Griffith, A. S., Report to Loc. Gov. Board, New Series, No. 88, Lond., 1914.

[Q. J. M., Oct., 1919.] 